\title{
E. coli and colon cancer: Is mutY a culprit?
}

\author{
Abdul Arif Khan ${ }^{1,2 *}$, Phillip Cash ${ }^{3}$ \\ ${ }^{1}$ Department of Microbiology, College of Life Sciences, Cancer Hospital \& Research \\ Institute, Gwalior, (MP) India \\ ${ }^{2}$ Department of Pharmaceutics, College of Pharmacy, PO Box 2457, King Saud University, \\ Riyadh 11451, Saudi Arabia \\ ${ }^{3}$ Division of Applied Medicine, School of Medicine, University of Aberdeen, Foresterhill, \\ Aberdeen AB25 2ZD, Scotland \\ *For correspondence: Department of Pharmaceutics, College of Pharmacy, King Saud \\ University, Riyadh 11451, Saudi Arabia, Email: abdularifkhan@gmail.com
}

\begin{abstract}
:
The recent demonstration of a role of $E$. coli in the development of invasive carcinoma in mice ushers a new era of bacterial involvement in cancer etiology. It has been shown previously that the colonic mucosa of colorectal carcinoma (CRC) is exclusively colonized by intracellular $E$. coli instead of extracellular form found in normal colonic mucosa. Surprisingly, the DNA repair gene MUTYH, which is a homologue of the $E$. coli gene mutY, is responsible for CRC. The current paper discusses the potential role of mutY in CRC etiology and concludes that research in this area can bring together the diverse threads of the CRC etiology puzzle.
\end{abstract}

Keywords: E. coli, mutY, MUTYH, chronic inflammation, carcinogenesis

\section{Introduction}


The enigmatic relationship of E. coli with colorectal cancer (CRC) has always raised suspicions for cancer researchers in the role of this bacterium in cancer development. Recently, an article published in the August 2012 issue of Science revealed that E. coli has the capability to promote invasive carcinoma in mice [1]. Although verification of its etiological potential in humans is still awaiting a legitimate appraisal, a logical mechanistic explanation for this association has been provided through chronic inflammation. Induction of proinflammatory cytokines and highly reactive chemical species during chronic inflammation leads to oxidation, nitration and chlorination of DNA, RNA and proteins. [2]. Khan et al suggested that the frequent association of $E$. coli with CRC may allow the bacteria to serve as an indicator of cancer development [3]. E. coli infection is involved in chronic inflammation of the intestine during inflammatory bowel disease (IBD). It has been observed that the pathogenesis of IBD involves a consistent increase in mucosa-associated E. coli with an "adherent and invasive" phenotype. Although many other bacterial species are also involved in IBD [4], the recent demonstration of E. coli induced carcinogenesis in mice attracts our attention towards this organism as a possible CRC etiologic agent in humans. Two forms of IBD, including Crohn's disease (CD) and ulcerative colitis (UC), are associated with an increased risk in the development of colon cancer [5,6].

\section{E. coli: a host cell cycle modulator}

Several studies have considered the potential role of microbes in CRC etiology [7,8]. A role for E. coli in CRC etiology gained much interest after finding mounting evidence for this association [9]. It has been verified in many studies that E. coli has an enormous potential for cell cycle modulation, thus increasing the chances for the development of cancer. Some $E$. coli strains produce cyclomodulins which interfere with the host cell cycle and may serve as a possible link for the association with E. coli and CRC [10]. In addition, cytotoxic necrotizing factor (CNF) is a cyclomodulin produced by E. coli; CNF activates rho GTP binding protein 
and prevents apoptosis. CNF modulates both mitochondrial homeostasis and the expression of Bcl-2 member of apoptotic regulators. It acts as an apoptosis inhibitor and cell cycle stimulator due to the induction of DNA replication and G1/S transition [11-13]. Another E. coli cyclomodulin, known as cycle inhibiting factor (Cif), has the ability to cause irreversible arrest of the cell cycle at the G2/M transition [14]. Cif, which is produced by enteropathogenic (EPEC) and enterohaemorrhagic (EHEC) E. coli strains [15], activates G2/M arrest by activating a DNA damage independent pathway through sustained inhibitory phosphorylation of mitosis inducer CDK1 [14]. Cif also causes unique alterations in the actin cytoskeleton leading to its anchoring to the host cell membrane and cellular and nuclear enlargement. Nuclear enlargement occurs due to continuous DNA replication in the absence of nuclear division [16]. This endoreduplication increases cellular DNA content and also increases gene copy number and proteins including growth factors leading to rapid growth of cells and possibly cancer $[13,17,18]$. Moreover, E. coli also produces cytolethal distending toxin (CDT), which causes cell cycle arrest by inflicting DNA damage [19,20]. CDT is a tripartite complex with the CdtB subunit homologous to human DNAse I [21]. The role of CDT in the carcinogenic process has been investigated in a microbial induced hepatocarcinogenesis mouse model. In this model it was observed that Helicobacter hepaticus CDT promoted the development of dysplasia from hepatitis and increased proliferation of hepatocytes [22].

It has been reported recently that host cell mismatch repair activity of E. coli infected cells is reduced in colon cancer cell lines as well as CRC patients [23]. A reduction of DNA repair activity is directly related to an accumulation of mutations and is a suspected reason for $E$. coli driven cancer. Besides these attributes of cell metabolism in E. coli induced cancer etiology, this bacteria have little or no capacity for the conversion of N-hydroxy-4-acetylaminobiphenyl (N-OH-AABP) to 4-acetylaminobiphenyl, which reverses activation of the 
parent carcinogen in the gut [24]. It has been known for some time that the gut microflora have the capacity to convert several carcinogens to harmless products in the intestine and thus

minimize the cancer risk [25]. However, during IBD E. coli is generally present as a prominent organism and can reduce this attribute of the gut microflora. Although many pieces of evidence support a proposed carcinogenic activity of E. coli, a satisfactory consensus cannot be achieved on any single mechanism. This situation demands the detection of firm evidence and the identification of specific mechanism for the E. coli-CRC association.

\section{E. coli and CRC: a peculiar association}

In addition to the mechanistic link between chronic inflammation and CRC development, it is also worthy of note that the colorectal carcinoma mucosa, but not normal colonic mucosa, is colonised by intracellular E. coli [26]. Chronic inflammation and consequent tissue damage induces a cascade of pro-inflammatory and anti-inflammatory molecules. The equilibrium between these two groups of regulators controls cell death and repair of tissue damage caused due to cell death. However, any problems with this equilibrium may lead to the development of cancer [27]. Cellular mutations are additional factors in regulating the development of CRC. Several cellular mutations have been identified as etiologic factors for colon cancer and these have been reviewed in many recent articles [28]. Among the mutations linked to CRC, MUTYH, which encodes for DNA glycosylase, is one of a number of genes involved in regulating oxidative DNA damage repair [29]. Although germ line mutations in MUTYH are linked to hereditary CRC, somatic mutations in this gene are also associated with cases of CRC [30]. It is noteworthy that the cellular MUTYH gene is a homologue of the E. coli gene mutY, which is also responsible for regulating DNA damage repair [29]. Thus it offers an additional target for investigating the E. coli-CRC association. 


\section{Possible role for the involvement of mutY in CRC etiology}

Considering the diverse lines of evidence described above for the microbial etiology of CRC, it is interesting to consider a potential role for the E. coli mutY gene in the development of colon cancer. E. coli can exist as a chronic intracellular pathogen in colon cells and this intimate association between the bacteria and colon cells provides an opportunity for the bacteria to grow for many generations and for the old bacterial cells to release their contents into the colon cell cytoplasm. Thus, we can speculate that the intracellular E. coli will release their DNA and mutY gene products into the colon cell.

This hypothesis raises three interesting questions. First, due to the high degree of homology between mutY and MUTYH, can horizontal gene transfer (HGT) events take place, which might affect DNA glycosylase performance? Second, can mutY gene products interfere with the activity of human MUTYH gene products? Third, do epigenetic factors that regulate mutY gene expression also influence MUTYH expression? Although remote, each of these three events could lead to an abnormal DNA repair pathway and ultimately promote the development of colon cancer (Figure 1).

\subsection{Horizontal gene transfer of mutY and CRC}

Indirect support for the involvement of HGT in CRC progression comes from the demonstration of extensive horizontal gene transfer from intracellular bacterial pathogens to their multi-cellular eukaryotic hosts. For example the intracellular bacterial endosymbiont, Wolbachia pipientis, has the ability to transfer genes horizontally to its host [31]. The intracellular residence of $E$. coli within colon cells during CRC may thus lead to a similar horizontal gene transfer [26]. Furthermore, several bacteria, particularly intracellular bacterial pathogens, can influence host metabolism and gene expression in order to sustain them within the host cellular environment. Several reports have been published on the subversion of host 
cellular responses by intracellular pathogens [32]. There is some skepticism about the transfer of genetic material between intracellular bacteria and the eukaryotic host cell due to the physical barriers that exist between the intracellular bacterium and host cell. However, in one comparative genomic study approximately 40 genes were identified that were consistent with lateral transfer between bacteria and humans [31]. During intracellular bacterial infection, the bacterial DNA acts as a symbiotic DNA molecule similar to mitochondrial or chloroplast DNA. It is reasonable to propose that these bacterial DNA fragments can affect the host MUTYH through horizontal gene transfer (HGT). The mechanism of HGT from bacteria in cancer etiology has been reviewed in a recent article [32]. However, it can be argued that MUTYH mutations are generally bi-allelic whereas E. coli induced events lead primarily to mono-allelic mutations. It is relevant to note that bi-allelic mutations are 10 times more prone to result in CRC compared to mono allelic mutations. Thus, bi- and mono-allelic mutations could be responsible for early and delayed onset cancers respectively [33].

\subsection{Direct effects of mutY protein or its epigenetic regulators and CRC}

Due to the high degree of homology between the bacterial mutY and eukaryotic MUTYH genes, both functional and structural analogies between the gene products are likely. It has been observed previously that the same enzymes from different organisms can differ in their degree of substrate specificity. This phenomenon is evident even for equivalent enzymes isolated from different bacteria. In the case of the equivalent enzyme from humans and $E$. coli, which are evolutionary distant, it is reasonable to propose that the presence of both enzymes in the same cell at one time can interfere with each other's activity. This latter scenario raises the possibility that DNA repair can be impaired, allowing the accumulation of mutations that ultimately lead to CRC. As discussed earlier, E. coli has the ability to reduce host cell DNA mismatch repair (MMR) in colonic cell lines and this has also been observed in CRC patients. This process has been suggested as a possible mechanism for E. coli 
induced colon carcinogenesis [23]. Enteropathogenic E. coli (EPEC) has been shown to secrete an effector protein (EspF), which is responsible for the reduction of MMR proteins [34]. However, the role of mutY in reducing the DNA repair activity of E. coli infected eukaryotic cells also requires a detailed appraisal.

It is relevant to note that microbes posses the ability to carry out epigenetic alterations of their host eukaryotic cells and induce pathological changes through epigenetic reprogramming [35]. Epigenetic changes leading to cancer include DNA methylation, histone modification and modulation of micro RNA expression [36,37]. Consequently, based on their sequence homology, mutY and MUTYH could share similar epigenetic regulators and thus cause comparable epigenetic alterations in the host cell DNA repair machinery. Micro RNAs are small RNA molecules involved in translation inhibition and silencing of many genes including those responsible for cell proliferation, differentiation and cell death. Gut microbiota have the capacity to modulate micro RNA expression thus influencing several biological processes in the host cell as recently reviewed by Masotti [38]. E. coli is a gram negative bacterium within the gut microbiota and has the potential to modulate the expression of micro RNA through it lipopolysaccharide (LPS). In macrophages, the LPS of gram negative bacteria can induce the expression of mir-155 but down regulate the expression of mir-125b micro RNAs [39]. The resident colonic microflora is responsible for the production of short chain fatty acids (SCFA) through fermentation of non digestible carbohydrates; these SCFAs include butyrate that is an inhibitor of histone deacetylase enzyme and can affect histone modification and DNA methylation [40,41]. DNA methylation is known to affect the expression of several cancer associated genes [42]. Since the pattern of DNA methylation varies between different organisms, it is possible that the presence of $E$. coli epigenetic regulators can lead to reprogramming thus altering the expression of host cell genes including MUTYH. Altered patterns of DNA methylation is a common molecular alteration observed 
in human cancers including CRC [43]. This scenario has already been described during epigenetic reprogramming of host genes by viruses and intracellular bacteria [44].

It is well-documented that enteropathogenic E. coli (EPEC) synthesise a variety of effector molecules that are capable of influencing cellular DNA repair [34]. The cytosolic localization of $E$. coli within colon cancer cells allows the bacteria to release the effector molecules directly into the host cell cytoplasm, although to affect host cellular processes these bacterial effector molecules must enter in the host cell nucleus. E. coli generally uses a Type III Secretion System (T3SS) to transfer the bacterial effector molecules into the eukaryotic host cell. However, when the bacteria are residing intracellularly the nuclear localization of the bacterial proteins depends upon the presence of nuclear localization signals (NLS) on the bacterial proteins [45]. Nuclear localization signals are required for the active transport of proteins from the cytoplasm to nucleus, although proteins with molecular weights $<40 \mathrm{kDa}$ can transfer to the nucleus via a passive transport mechanism $[45,46]$. Surprisingly, the mutY protein (NCBI Accession number EGT70329) has a molecular weight of $39.18 \mathrm{kDa}$ giving strong support for its nuclear localization via the passive transport mechanism. Similar to the possible mutY nuclear transport, the epigenetic regulators have a high propensity for an intracellular localization due to functional relatedness. For example, E. coli replication terminator protein Tus, an E. coli epigenetic regulator, has both a NLS and nuclear export signal (NES), and can therefore target the host cell nucleus. Although research in this area is in the early days, Tus protein was one of the first proteins shown to possess both NLS and NES, thus supporting a role in epigenetic targeting of the host cell nucleus [47]. However, future research on bacterial epigenetic regulators will, no doubt, identify similar host nuclear targeting potential for many E. coli proteins, perhaps including mutY. Therefore, chronic E. coli infection may create a situation where chronic inflammation, caused by the E. coli 
infection, leads to mutY mediated cellular alterations in the host cell DNA repair machinery that controls the development of CRC.

\section{Concluding remarks}

Our current understanding of CRC pathogenesis raises suspicion for a potential role of $E$. coli mutY in colon cancer and research in this direction is uncovering several aspects of CRC pathogenesis. Further research is required to address the potential role of E. coli in colon cancer development. Several aspects of the proposal outlined above, specifically the intracellular growth of $E$. coli in colon cancer cells, the homology between the mutY and MUTYH genes, possible horizontal gene transfer from the intracellular E. coli during colon cancer, and the presence of mutY and MUTYH gene products in same cell provide clues for linking these threads. Further research will provide new avenues to understand CRC etiology and help us to plan and implement infection control measures to control CRC. Managing an infection is much easier than managing the cancer itself; hence the role of E. coli mutY in CRC should be investigated further.

Conflict of Interest: Authors do not have any potential conflict of interest related to this work.

\section{References}

(1) J.C. Arthur, E. Perez-Chanona, M. Muhlbauer, S. Tomkovich, J.M. Uronis, T.J. Fan, B.J. Campbell, T. Abujamel, B. Dogan, A.B. Rogers, J.M. Rhodes, A. Stintzi, K.W. Simpson, J.J. Hansen, T.O. Keku, A.A. Fodor, C. Jobin. Intestinal Inflammation Targets Cancer-Inducing Activity of the Microbiota. Science 338 (2012) 120-3.

(2) G.N. Wogan, P.C. Dedon, S.R. Tannenbaum, J.G. Fox. Infection, inflammation and colon carcinogenesis. Oncotarget 3 (2012) 737-8. 
(3) A.A. Khan, A. Shrivastava, M. Khurshid. Normal to cancer microbiome transformation and its implication in cancer diagnosis. Biochim Biophys Acta 1826 (2012) 331-7.

(4) M. Friswell, B. Campbell, J. Rhodes. The role of bacteria in the pathogenesis of inflammatory bowel disease. Gut Liver 4 (2010) 295-306.

(5) A. Ekbom, C. Helmick, M. Zack, H.O. Adami. Ulcerative colitis and colorectal cancer. A population-based study. N Engl J Med 323 (1990) 1228-33.

(6) C.D. Gillen, H.A. Andrews, P. Prior, R.N. Allan. Crohn's disease and colorectal cancer. Gut 35 (1994) 651-5.

(7) D. Collins, A.M. Hogan, D.C. Winter. Microbial and viral pathogens in colorectal cancer. Lancet Oncol 12 (2011) 504-12.

(8) V. Antonic, A. Stojadinovic, K.E. Kester, P.J. Weina, B.L. Brucher, M. Protic, I. Avital, M. Izadjoo. Significance of infectious agents in colorectal cancer development. J Cancer 4 (2013) $227-40$.

(9) H.M. Martin, B.J. Campbell, C.A. Hart, C. Mpofu, M. Nayar, R. Singh, H. Englyst, H.F. Williams, J.M. Rhodes. Enhanced Escherichia coli adherence and invasion in Crohn's disease and colon cancer. Gastroenterology 127 (2004) 80-93.

(10) E. Buc, D. Dubois, P. Sauvanet, J. Raisch, J. Delmas, A. Darfeuille-Michaud, D. Pezet, R. Bonnet. High prevalence of mucosa-associated E. coli producing cyclomodulin and genotoxin in colon cancer. PLoS One 8 (2013) e56964.

(11) C. Fiorentini, P. Matarrese, E. Straface, L. Falzano, A. Fabbri, G. Donelli, A. Cossarizza, P. Boquet, W. Malorni. Toxin-induced activation of Rho GTP-binding protein increases Bcl-2 expression and influences mitochondrial homeostasis. Exp Cell Res 242 (1998) 341-50.

(12) E. Oswald, M. Sugai, A. Labigne, H.C. Wu, C. Fiorentini, P. Boquet, A.D. O'Brien. Cytotoxic necrotizing factor type 2 produced by virulent Escherichia coli modifies the small GTPbinding proteins Rho involved in assembly of actin stress fibers. Proc Natl Acad Sci U S A 91 (1994) 3814-8. 
(13) D.L. Mager. Bacteria and cancer: cause, coincidence or cure? A review. J Transl Med 4 (2006) 14.

(14) F. Taieb, J.P. Nougayrede, C. Watrin, A. Samba-Louaka, E. Oswald. Escherichia coli cyclomodulin Cif induces G2 arrest of the host cell cycle without activation of the DNAdamage checkpoint-signalling pathway. Cell Microbiol 8 (2006) 1910-21.

(15) O. Marches, T.N. Ledger, M. Boury, M. Ohara, X. Tu, F. Goffaux, J. Mainil, I. Rosenshine, M. Sugai, J. De Rycke, E. Oswald. Enteropathogenic and enterohaemorrhagic Escherichia coli deliver a novel effector called Cif, which blocks cell cycle G2/M transition. Mol Microbiol 50 (2003) 1553-67.

(16) J.P. Nougayrede, F. Taieb, J. De Rycke, E. Oswald. Cyclomodulins: bacterial effectors that modulate the eukaryotic cell cycle. Trends Microbiol 13 (2005) 103-10.

(17) B.A. Edgar, T.L. Orr-Weaver. Endoreplication cell cycles: more for less. Cell 105 (2001) 297306.

(18) X. Charpentier, E. Oswald. Identification of the secretion and translocation domain of the enteropathogenic and enterohemorrhagic Escherichia coli effector Cif, using TEM-1 betalactamase as a new fluorescence-based reporter. J Bacteriol 186 (2004) 5486-95.

(19) E. Haghjoo, J.E. Galan. Salmonella typhi encodes a functional cytolethal distending toxin that is delivered into host cells by a bacterial-internalization pathway. Proc Natl Acad Sci U S A 101 (2004) 4614-9.

(20) I. Toth, F. Herault, L. Beutin, E. Oswald. Production of cytolethal distending toxins by pathogenic Escherichia coli strains isolated from human and animal sources: establishment of the existence of a new cdt variant (Type IV). J Clin Microbiol 41 (2003) 4285-91.

(21) M. Lara-Tejero, J.E. Galan. CdtA, CdtB, and CdtC form a tripartite complex that is required for cytolethal distending toxin activity. Infect Immun 69 (2001) 4358-65. 
(22) Z. Ge, A.B. Rogers, Y. Feng, A. Lee, S. Xu, N.S. Taylor, J.G. Fox. Bacterial cytolethal distending toxin promotes the development of dysplasia in a model of microbially induced hepatocarcinogenesis. Cell Microbiol 9 (2007) 2070-80.

(23) O.D. Maddocks, A.J. Short, M.S. Donnenberg, S. Bader, D.J. Harrison. Attaching and effacing Escherichia coli downregulate DNA mismatch repair protein in vitro and are associated with colorectal adenocarcinomas in humans. PLoS One 4 (2009) e5517.

(24) L.A. Wheeler, F.B. Soderberg, P. Goldman. The reduction of N-hydroxy-4acetylaminobiphenyl by the intestinal microflora of the rat. Cancer Res 35 (1975) 2962-8.

(25) A.A. Khan, M. Khurshid, S. Khan, A. Alshamsan. Gut microbiota and probiotics: current status and their role in cancer therapeutics. Drug Dev Res doi: 10.1002/ddr.21087 (2013).

(26) A. Swidsinski, M. Khilkin, D. Kerjaschki, S. Schreiber, M. Ortner, J. Weber, H. Lochs. Association between intraepithelial Escherichia coli and colorectal cancer. Gastroenterology 115 (1998) 281-6.

(27) H. Lu, W. Ouyang, C. Huang. Inflammation, a key event in cancer development. Mol Cancer Res 4 (2006) 221-33.

(28) S.D. Markowitz, M.M. Bertagnolli. Molecular origins of cancer: Molecular basis of colorectal cancer. N Engl J Med 361 (2009) 2449-60.

(29) M.L. Poulsen, M.L. Bisgaard. MUTYH Associated Polyposis (MAP). Curr Genomics 9 (2008) 420-35.

(30) K. Bougatef, R. Marrakchi, N. Kourda, Y.B. Ben Lahely, S.B. Jileni, H.K. El Khil, F. Soubrier, A. Ben Ammar Elgaaied. Somatic mutation of MutYH in Tunisian patients with sporadic colorectal cancer. J Clin Lab Anal 21 (2007) 372-4.

(31) J.C. Dunning Hotopp, M.E. Clark, D.C. Oliveira, J.M. Foster, P. Fischer, M.C. Munoz Torres, J.D. Giebel, N. Kumar, N. Ishmael, S. Wang, J. Ingram, R.V. Nene, J. Shepard, J. Tomkins, S. Richards, D.J. Spiro, E. Ghedin, B.E. Slatko, H. Tettelin, J.H. Werren. Widespread lateral gene transfer from intracellular bacteria to multicellular eukaryotes. Science 317 (2007) 1753-6. 
(32) Editorial. Focus on host subversion. Nat Cell Biol 11 (2009) 509.

(33) M.A. Jenkins, M.E. Croitoru, N. Monga, S.P. Cleary, M. Cotterchio, J.L. Hopper, S. Gallinger. Risk of colorectal cancer in monoallelic and biallelic carriers of MYH mutations: a populationbased case-family study. Cancer Epidemiol Biomarkers Prev 15 (2006) 312-4.

(34) O.D. Maddocks, K.M. Scanlon, M.S. Donnenberg. An Escherichia coli Effector Protein Promotes Host Mutation via Depletion of DNA Mismatch Repair Proteins. MBio 4 (2013).

(35) J. Minarovits. Microbe-induced epigenetic alterations in host cells: the coming era of pathoepigenetics of microbial infections. A review. Acta Microbiol Immunol Hung 56 (2009) 1-19.

(36) K. Gronbaek, C. Hother, P.A. Jones. Epigenetic changes in cancer. Apmis 115 (2007) 1039-59.

(37) S. Sassen, E.A. Miska, C. Caldas. MicroRNA: implications for cancer. Virchows Arch 452 (2008) 1-10.

(38) A. Masotti. Interplays between gut microbiota and gene expression regulation by miRNAs. Front Cell Infect Microbiol 2 (2012) 137.

(39) E. Tili, J.J. Michaille, A. Cimino, S. Costinean, C.D. Dumitru, B. Adair, M. Fabbri, H. Alder, C.G. Liu, G.A. Calin, C.M. Croce. Modulation of miR-155 and miR-125b levels following lipopolysaccharide/TNF-alpha stimulation and their possible roles in regulating the response to endotoxin shock. J Immunol 179 (2007) 5082-9.

(40) K. Meijer, P. de Vos, M.G. Priebe. Butyrate and other short-chain fatty acids as modulators of immunity: what relevance for health? Curr Opin Clin Nutr Metab Care 13 (2010) 715-21.

(41) L. Conterno, F. Fava, R. Viola, K.M. Tuohy. Obesity and the gut microbiota: does upregulating colonic fermentation protect against obesity and metabolic disease? Genes Nutr 6 (2011) 241-60.

(42) S.B. Baylin, M. Esteller, M.R. Rountree, K.E. Bachman, K. Schuebel, J.G. Herman. Aberrant patterns of DNA methylation, chromatin formation and gene expression in cancer. Hum Mol Genet 10 (2001) 687-92. 
(43) M. Zitt, H.M. Muller. DNA methylation in colorectal cancer--impact on screening and therapy monitoring modalities? Dis Markers 23 (2007) 51-71.

(44) K. Paschos, M.J. Allday. Epigenetic reprogramming of host genes in viral and microbial pathogenesis. Trends Microbiol 18 (2010) 439-47.

(45) P. Dean. Functional domains and motifs of bacterial type III effector proteins and their roles in infection. FEMS Microbiol Rev 35 (2011) 1100-25.

(46) E.J. Tran, S.R. Wente. Dynamic nuclear pore complexes: life on the edge. Cell 125 (2006) 1041-53.

(47) S.J. Kaczmarczyk, K. Sitaraman, T. Hill, J.L. Hartley, D.K. Chatterjee. Tus, an E. coli protein, contains mammalian nuclear targeting and exporting signals. PLoS One 5 (2010) e8889. 

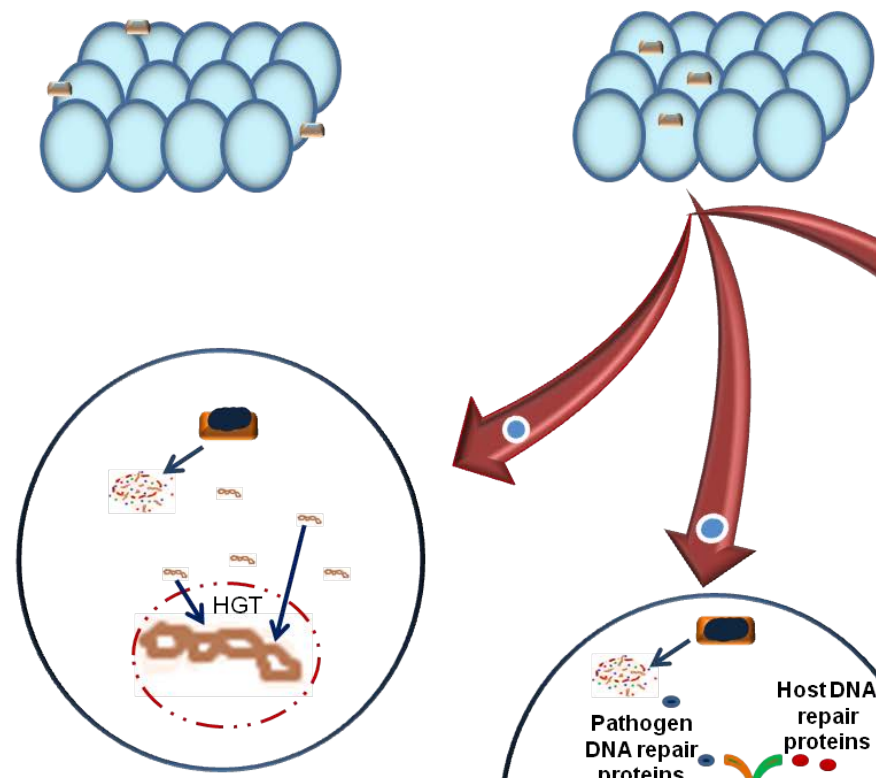

A

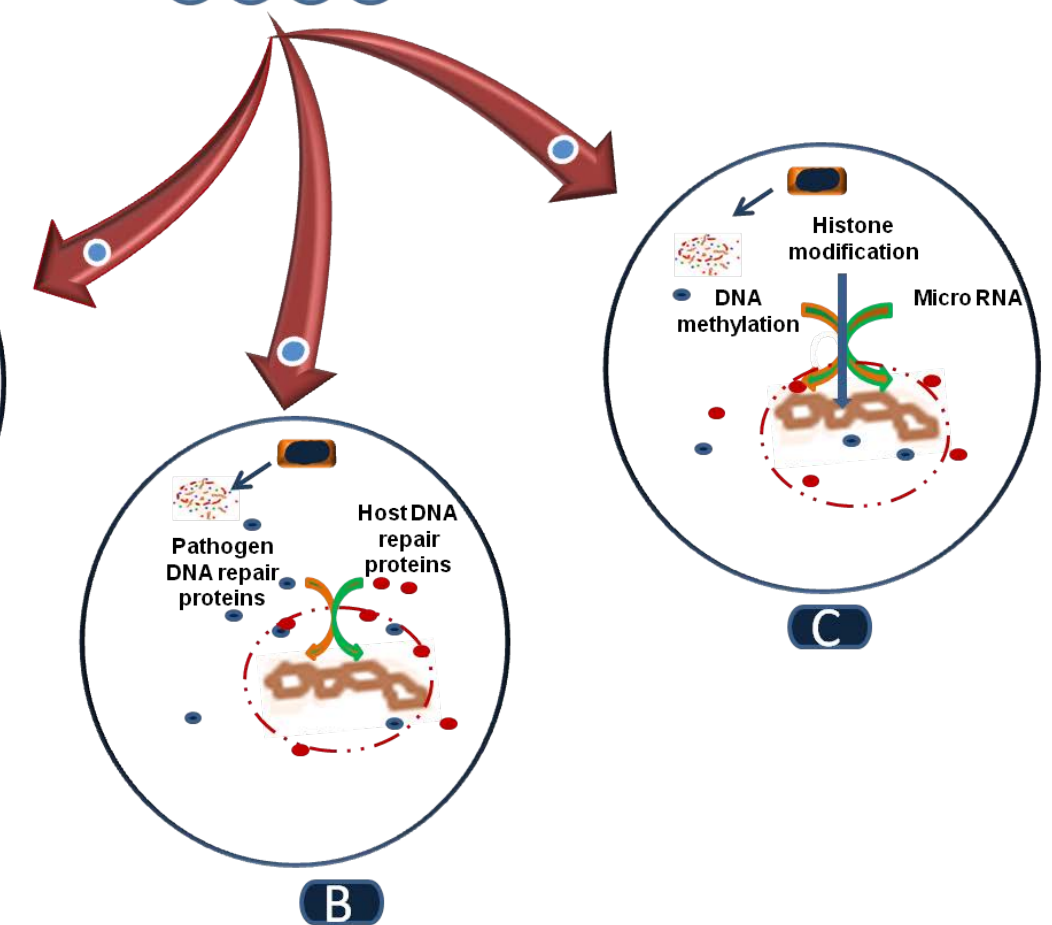

Figure 1: Proposed explanation for the potential involvement of E. coli mutY in CRC etiology. During chronic E. coli infection, the bacteria establish an intracellular residence of bacteria in associated colon cancer cells. (A) Shedding of the bacterial DNA into the host cell and due to sequence homology between mutY and MUTYH, horizontal gene transfer events may occur, which can lead to the development of CRC. (B) Products of E. coli mutY and human MUTYH can also affect the ability of DNA repair in host cell (C) Epigenetic regulators of the bacterial mutY gene can also affect human MUTYH leading to abnormal expression of MUTYH and ultimately the development of CRC. 